\title{
A surface defect versus microstructure under fatigue loading: Experimental and numerical approach
}

\author{
Matthieu Vincent ${ }^{\mathrm{a}}$, Yves Nadot, Carole Nadot-Martin and André Dragon \\ Institut Pprime, CNRS UPR 3346, ISAE-ENSMA, Université de Poitiers, Département Physique et \\ Mécanique des Matériaux, Téléport 2, 1 avenue Clément Ader, BP. 40109, 86961 Futuroscope \\ Chasseneuil Cedex, France
}

The fatigue design of metallic parts is strongly connected to the manufacturing (casting and manufacturing process) and the service loading (foreign object damage (FOD) and maintenance degradation). The designer needs to reach a compromise between the fatigue resistance of the component and the allowable defect due to the process. A defect can be considered against fatigue using the following parameters: type (inclusion, oxide, pore, shrinkage...), morphology (spherical, ellipsoidal, complex...), position (internal, sub surface or surface) and size. For a given material, Kitagawa diagram [1] (evolution of fatigue limit with defect size) shows two regimes: defect smaller than a critical size does not change fatigue limit, while defect larger than a critical size leads to decrease of fatigue limit.

The aim of this paper is to present a description of the influence of defect on the fatigue resistance for a material as dependent on its microstructure. The material for the experimental approach is pure iron, a 'school' material roughly isotropic regarding plasticity effects, using test specimens with controllable grain sizes [2] (Fig. 1). Experimental Wöhler curves (defect free and various defect sizes in Fig. 2) allow to interpret mechanisms of fatigue degradation. Further understanding of mechanisms is reached by analyzing the distribution of mechanical fields by Finite Element simulation of truncated hexahedron regular microstructure with a surface defect (Fig. 3) [3-5].

\footnotetext{
${ }^{a}$ Corresponding author: matthieu.vincent@ensma.fr

This is an Open Access article distributed under the terms of the Creative Commons Attribution License 4.0, which permits unrestricted use, distribution, and reproduction in any medium, provided the original work is properly cited.
} 


\section{MATEC Web of Conferences}

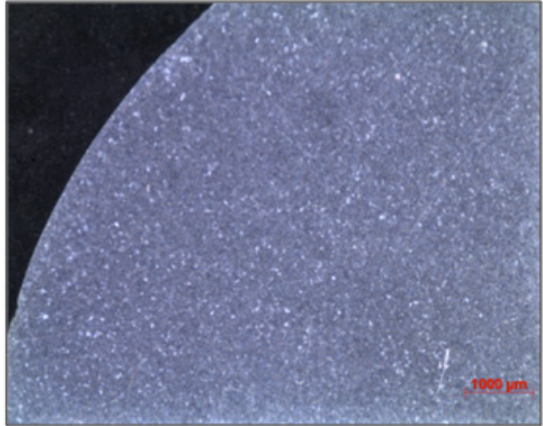

(a)

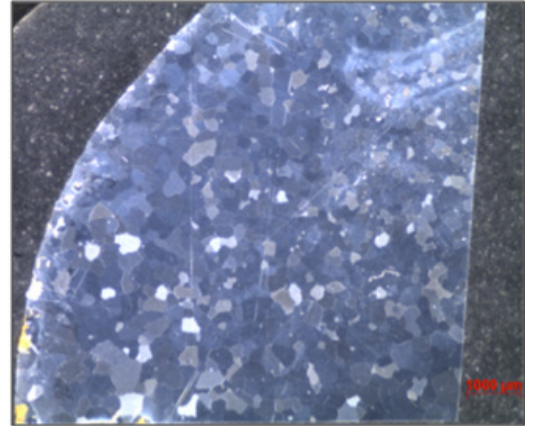

(b)

Figure 1. ARMCO iron microstructure: (a) no treatment; (b) cold-rolled $10 \%, 150 \mathrm{~h}$ at $850^{\circ} \mathrm{C}$.

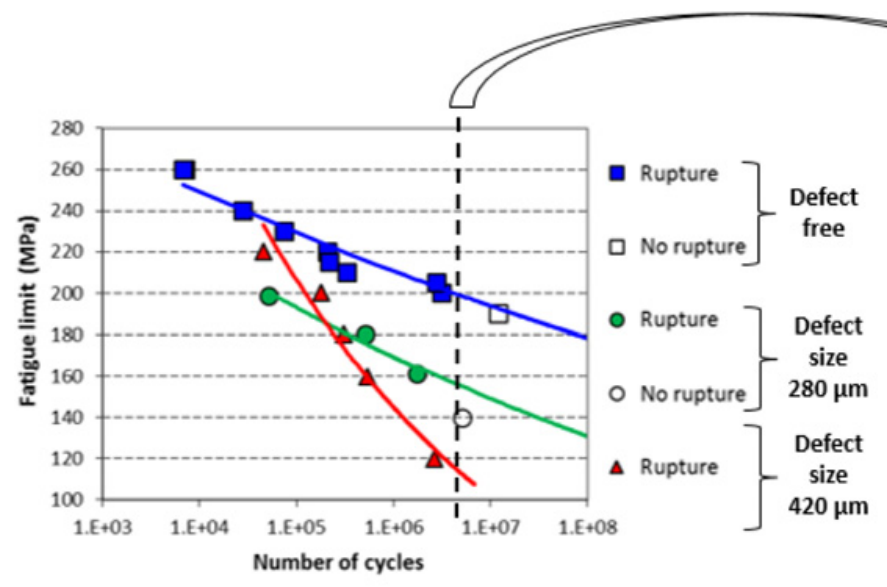

(a)

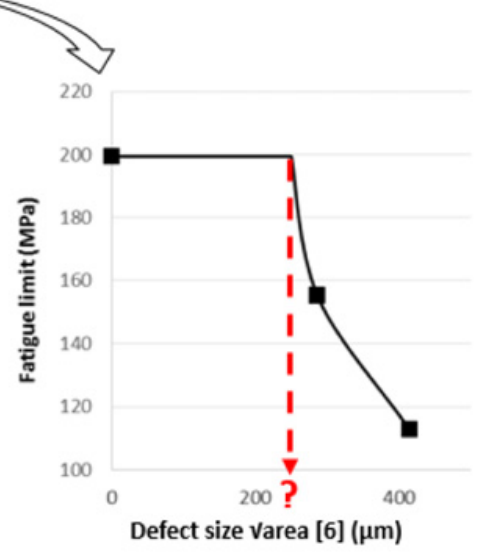

(b)

Figure 2. Experimental results obtained with ARMCO iron without treatment: (a) Wöhler curves; (b) Kitagawa diagram at 5 million cycles.

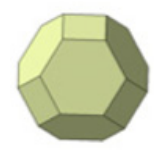

(a)

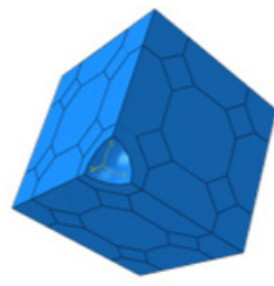

(b)

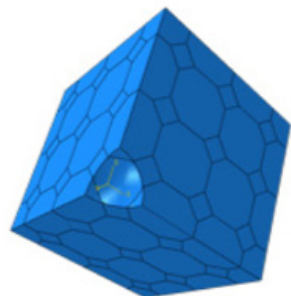

(c)

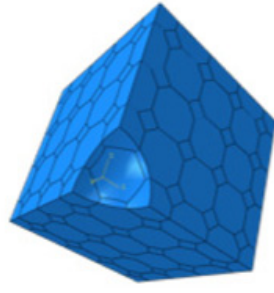

(d)

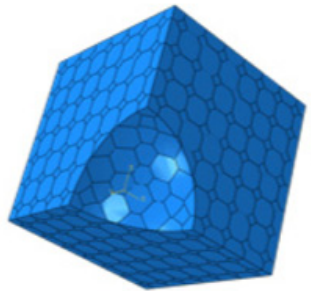

(e)

Figure 3. Truncated hexahedron regular microstructure (a) with a surface defect: defect diameter smaller than a grain size (b), equal to grain size (c), equal to twice the grain size (d) and equal to six times the grain size (e). 


\section{FDMDII - JIP 2014}

\section{References}

[1] H. Kitagawa, S. Takahashi, Int. Conf. on Mech. Behaviour of Mat., Boston, 627-631 (1976)

[2] R. Pippan, Mat. Sc. and Eng. 138, 1-13 (1991)

[3] F. Barbe, L. Decker, D. Jeulin, G. Cailletaud, Int. J. of Plasticity 4, 513-536 (2001)

[4] Y. Guilhem, S. Basseville, F. Curtit, J-M. Stéphan, G. Cailletaud, Int. J. of Fatigue 32, 1748-1763 (2010)

[5] M. Sauzay, T. Jourdan, Int. J. of Fracture 141, 431-446 (2006)

[6] Y. Murakami, Metal Fatigue: Effects of Small Defects and Nonmetallic Inclusions (Elsevier Science Ltd, Oxford, 2002) 